\title{
A New Approach of In Vivo Musculoskeletal Tissue Engineering Using the Epigastric Artery as Central Core Vessel of a 3-Dimensional Construct
}

\author{
Sebastian E. Dunda, ${ }^{1}$ T. Schriever, ${ }^{1}$ C. Rosen, ${ }^{2}$ C. Opländer, ${ }^{1}$ R. H. Tolba, ${ }^{3}$ S. Diamantouros, ${ }^{4}$ \\ S. Jockenhoevel, ${ }^{4}$ and N. Pallua ${ }^{1}$ \\ ${ }^{1}$ Department of Plastic Surgery, Reconstructive and Hand Surgery, Burn Center, University Hospital Aachen, RWTH, \\ Pauwelsstraße 30, 52074 Aachen, Germany \\ ${ }^{2}$ Department of Anatomy, University Hospital Aachen, 52074 Aachen, Germany \\ ${ }^{3}$ Institute of Laboratory Animal Science and Experimental Surgery, University Hospital Aachen, 52074 Aachen, Germany \\ ${ }^{4}$ Helmholtz Institute for Biomedical Engineering, RWTH Aachen, 52074 Aachen, Germany
}

Correspondence should be addressed to Sebastian E. Dunda, sdunda@ukaachen.de

Received 18 September 2011; Accepted 5 December 2011

Academic Editor: Jan-Thorsten Schantz

Copyright (C) 2012 Sebastian E. Dunda et al. This is an open access article distributed under the Creative Commons Attribution License, which permits unrestricted use, distribution, and reproduction in any medium, provided the original work is properly cited.

\begin{abstract}
The creation of musculoskeletal tissue represents an alternative for the replacement of soft tissue in reconstructive surgery. However, most of the approaches of creating artificial tissue have their limitations in the size as the maximally obtainable dimension of bioartificial tissue (BAT) is limited due to the lack of supporting vessels within the 3-dimensional construct. The seeded myoblasts require high amounts of perfusion, oxygen, and nutrients to survive. To achieve this, we developed a 3-dimensional scaffold which features the epigastric artery as macroscopic core vessel inside the BAT in a rat model (perfused group, $n=4$ ) and a control group $(n=3)$ without the epigastric vessels and, therefore, without perfusion. The in vivo monitoring of the transplanted myoblasts was assessed by bioluminescence imaging and showed both the viability of the epigastric artery within the 3-dimensional construct and again that cell survival in vivo is highly depending on the blood supply with the beginning of capillarization within the BAT seven days after transplantation in the perfused group. However, further studies focussing on the matrix improvement will be necessary to create a transplantable BAT with the epigastric artery as anastomosable vessel.
\end{abstract}

\section{Introduction}

The replacement and reconstruction of musculoskeletal tissue after severe damage caused by traumatic injury, tumor surgery, or prolonged denervation is limited with the utilizable number of transplantable muscle tissue. Moreover, the transfer of muscle tissue is not uncommonly associated with aesthetic and functional impairment at the donor site. Thus, musculoskeletal tissue engineering is attempting to be an alternative in the challenging field of reconstructive surgery [1-5]. However, during the last decade of stem cell and tissue engineering research, it turned out that one main critical factor in tissue engineering is the lack of supporting blood vessels and insufficient capillarization [6,7]. Therefore different in vivo models like the AV-loop using the femoral vessels have been developed and confirmed a much higher cell survival by inducing vascularisation of the used matrix and furthermore the possibility to create a 3D-bioartificial tissue construct [8-14]. In this study, we established a new in vivo model of musculoskeletal tissue engineering using the inferior and superior epigastric artery as central-core vessels of our custom-made implantable bioreactor chamber. In order to follow-up our transplanted myoblasts in vivo, the cells have been transfected with Luciferase and could in this way be atraumatically monitored with bioluminescence [15-17]. 


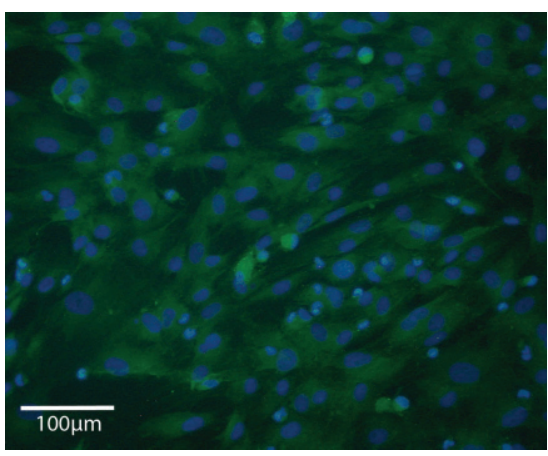

FIGURE 1: Immunofluorescence, 200x. 2D-culture of myoblasts harvested from 5 days old male Wistar rats: Desmin (green), nucleic counterstain with DAPI (blue).

\section{Materials and Methods}

2.1. Myoblasts Cell Culture. Myoblasts were harvested by dissecting the soleus and gastrocnemius muscles of 5 days old male Wistar rats (Charles River, Kisslegg, Germany) as described by Bach et al. [12]. Satellite cells were obtained from the minced muscles by digestion with $0.1 \%$ collagenase type III (Biochrom, Berlin, Germany) for 60 minutes and $0.25 \%$ trypsin (Viralex, PAA Laboratories, Linz, Austria) for 45 minutes at $37^{\circ}$ Celsius. These cells were filtered and cell culture was performed in Dulbecco's modified Eagle's medium containing $1 \%$ penicillin/streptomycin solution and $10 \%$ foetal bovine serum (all from FBS, Viralex, PAA Laboratories, Linz, Austria). Medium was changed every 2 to 3 days and cells were expanded through two passages by detachment using $0.25 \%$ trypsin, followed by resuspension, collection, and seeding in new culture flasks at a ratio of $1: 3$.

2.2. Immunofluorescence Staining for Desmin. The myogenic specificity of harvested myoblasts was verified by Desminand MyoD-immunocytochemistry (see Figure 1 (Desmin) before transplantation and Figure 6 (MyoD) after explantation). Therefore, the cell cultures were fixed in $100 \%$ methanol for 20 minutes at $-20^{\circ}$ Celsius, washed with (PBS Biochrom, Berlin, Germany), and permeabilized with TRITON X-100 (Sigma-Aldrich, Irvine, UK) 0.25\% in PBS for 30 minutes. The cultures were then incubated with blocking solution (5\% goat serum and $0.1 \%$ TRITON X-100 in PBS) for 30 minutes. After being washed with PBS, the cultures were incubated with rabbit anti-Desmin polyclonal antibody (Sigma-Aldrich, Irvine, UK). The cultures were then washed with PBS and incubated with anti-rabbit, IgG for 2 hours diluted $1: 1500$ in blocking solution. Finally nuclear counterstaining with DAPI (Sigma Aldrich, Irvine, UK) was performed through incubation with DAPI $10 \mu \mathrm{g} / 1 \mathrm{~mL}$ aqua bidest for 30 minutes at room temperature followed by washing steps. After being mounted with Mowiol (Sigma-Aldrich, Irvine, UK), the Desmin-stained cultures were viewed and photographed by fluorescence microscopy (Carl Zeiss MicroImaging, Jena, Germany).

2.3. Lentiviral Production and Infection (Luciferase). For the production of lentivirus, $7 \times 10^{6} \mathrm{HEK} 293 \mathrm{~T}$ cells were

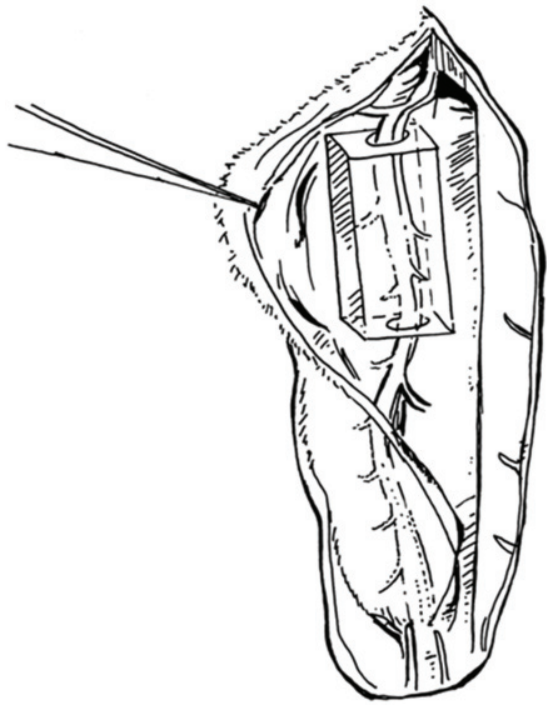

FIGURE 2: Implantation site showing the rectus abdominis dissected free with the superior and inferior epigastric vascular pedicle along the deep surface of the muscle. The in vivo bioreactor chamber is assembled around the epigastric vessels.

seeded onto a $15 \mathrm{~cm}$ Petri dish and transfected after $24 \mathrm{~h}$ with jetPEI (PEQLAB) using 9.4 g of transfer vector pEMWLuc (containing the coding sequence of Luciferase), $6.1 \mathrm{~g}$ of psPAX2 (packaging plasmid), and $3.3 \mathrm{~g}$ of pMD2. G (VSV$\mathrm{G}$ envelope). Lentivirus were harvested $48 \mathrm{~h}$ after transfection, passed through a $0.22 \mu \mathrm{m}$ filter, and concentrated by ultracentrifugation at $26000 \mathrm{rpm}$ for $2 \mathrm{~h}$ at $4^{\circ} \mathrm{C}$. Viral particles were resuspended in PBS and stored at $-80^{\circ} \mathrm{C}$. For infection with the lentiviral stock, primary myoblasts were seeded in $10-\mathrm{cm}$ plates in complete medium and infected with viral particles (MOI 5) and $8 \mathrm{~g} / \mathrm{L}$ polybrene for $16 \mathrm{~h}$ at $37^{\circ} \mathrm{C}$. Media were changed to growth media without polybrene. The average percentage of success of the transfected myoblasts was 89.4 percent in our efficacy tests.

2.4. Surgical Procedure. Male Wistar rats (Charles River, Kisslegg, Germany) at the age of 4 to 5 weeks served as recipients of the in vivo bioreactor chamber. German regulations for the care and use of laboratory animals have been observed at all times. All experimental protocols were approved by the governmental review committee of Northrhine-Westfalia, Germany. The animals were housed in the veterinary care facility of the RWTH University Hospital under temperature-controlled conditions at $21 \pm 1^{\circ} \mathrm{C}$ with a cycle of 12-hour light and 12-hour darkness as well as free access to standard food and water.

Operations were performed by the same microsurgeon under an operative microscope (Karl-Zeiss, Jena, Germany). Anesthesia was induced with inhalative Isoflurane (Baxter, Unterschleißheim, Germany) and maintained by intraperitoneal injection of Ketamine $(10 \%, 0.6 \mathrm{~mL} / \mathrm{kg})$ and Medetomidine $(0.3 \mathrm{~mL} / \mathrm{kg}$; Pfizer, Paris, France). Ketoprofen $(5 \mathrm{mg} / \mathrm{kg})$ was administered preoperatively for analgesia. The surgical site was shaved, prepped, and draped for sterility. A mid-line incision was made and perforating branches to the 
cutaneous tissue were cauterized. The dissection of the rectus abdominis muscle was performed along the linea alba as described by Zhang et al. [18] starting retrograde from $2 \mathrm{~cm}$ above the symphysis pubis to the xyphoid (see Figure 2). Furthermore, for the group with perfused BAT $(n=4)$, the epigastric artery and vein were dissected carefully from the rectus abdominis muscle with a total length of at least $3 \mathrm{~cm}$. The lower part of the polyoxymethylene (POM) customized bioreactor chamber (outer width $10 \mathrm{~mm}$, length $20 \mathrm{~mm}$, height $6 \mathrm{~mm}$ ) was positioned under the epigastric vessels using these vessels as central-core vessels of the in vivo chamber. Fibrin glue was administered as matrix (Baxter, Unterschleißheim, Germany) embedding the epigastric vessels in the bioreactor, and $4 \times 10^{6}$ myoblasts were suspended and mixed with the fibrin glue subsequently to complete the bioartificial tissue (see Figure 3). The bioreactor chamber was closed by attaching the upper part. The blood flow of the epigastric artery was checked by Doppler sonography proximal and distal of the chamber to ensure the patency before wound closing. Subsequently, the rectus abdominis muscle was adapted using Prolene 5-0 (Ethicon, Norderstedt, Germany) to achieve stability of the abdominal wall and protection of the bioreactor chamber. Finally, the wound was closed using Vicryl 5-0 (Ethicon, Norderstedt, Germany). In the control group with unperfused BAT $(n=3)$, the bioreactor chamber was implanted as described but without preparation and use of the epigastric vessels. For the explantation of the bioreactor chamber, the animals were reanaesthetized as described before on day seven, and after exposing the chamber, the epigastric artery was ligated and transected proximal and distal of the chamber allowing to remove the bioreactor completely without manipulation. After this explantation on day seven, the animals were sacrificed by intracardiac potassium chloride injections.

2.5. Bioluminescence Imaging. The followup of the transplanted myoblasts inside the in vivo bioreactor chamber was assessed by means of bioimaging with an ultrasensitive Xenogen camera (IVIS Lumina, Caliper Life Sciences, MA, USA) using the reporter D-Luciferin at different time points $(\mathrm{d} 0, \mathrm{~d} 1, \mathrm{~d} 7)$. The animals were anesthetized with $2 \%$ Isoflurane and placed in the imaging chamber. After acquisition of a baseline image, the rats were injected with the substrate Luciferin intraperitoneally in order to induce photons (green light emission at $562 \mathrm{~nm}$ ). The region of interest (ROI) was defined as the site where the in vivo bioreactor chamber is located. Peak signal (photons/second $[\mathrm{p} / \mathrm{s}]$ ) from that fixed ROI was evaluated using the Living Image 2.50 software (Xenogen). The background signal was evaluated at different locations not close to the bioreactor chamber as well as at different time points (d0, d1, d7) and subtracted from the corresponding peak signal.

2.6. Immunofluorescence Staining for MyoD (after Explantation). Cryosections of explanted bioartificial tissue containing myoblasts of $\mathrm{d} 7$ were washed with PBS. The cultures were then incubated with blocking solution (5\% goat serum and $0.1 \%$ TRITON X-100 in PBS) for 10 min. After being washed

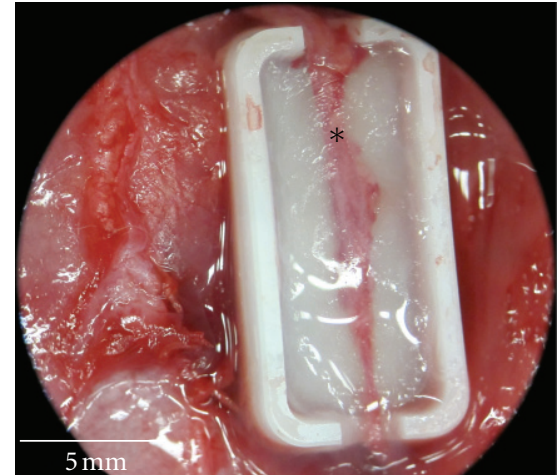

FIGURE 3: The implanted in vivo bioreactor chamber (outer width $10 \mathrm{~mm}$, length $20 \mathrm{~mm}$, height $6 \mathrm{~mm}$ ) in a 4-weeks-old male Wistar rat with the epigastric artery $(*)$ as central-core vessel; the transplanted and luciferase-transfected myoblasts are embedded in a fibrinogen matrix.

with PBS, the cultures were incubated with mouse antiMyoD monoclonal antibody (Thermo Scientific, Astmoor, $\mathrm{UK})$, diluted $1: 50$ in blocking solution $\left(2 \mathrm{~h}\right.$ at $\left.20^{\circ} \mathrm{C}\right)$, and again washed with $\mathrm{PBS}$ and incubated with anti-mouse $\operatorname{IgG}$ (KPL, MD, USA) for $2 \mathrm{~h}$ diluted $1: 100$ in blocking solution. After being washed with PBS and mounted with Mowiol, anti-MyoD-stained cultures were viewed and photographed by fluorescence microscopy.

\section{Results and Discussion}

3.1. Myoblasts Can Be Luciferase-Transfected and Therefore Monitored In Vivo with Bioluminescence. The bioluminescence followup monitoring of the Luciferase-transfected myoblasts showed the expected results with a high number of photons per second on day 0 directly after surgery $(1.769 \times$ $10^{8}$ photons/sec, see Figure 4 and Table 1$)$ and exponentially decreasing bioluminescence signals on day 1 and $7(3.426 \times$ $10^{7}$ photons $/ \mathrm{sec}$ and $3.031 \times 10^{6}$ photons $/ \mathrm{sec}$ ) indicating the remission of the myoblasts cell survival. The evaluated background signals $\left(4.822 \times 10^{5}\right.$ photons/sec $)$ showed no significant differences in terms of location and time points. However, in unperfused BATs without arterial inflow, and therefore, no possible Luciferin supply, only the background signals (e.g., $4.110 \times 10^{5}$ photons/sec on day 0 ) could be detected as well. Furthermore, the circumstance of retrieving bioluminescence signals in the perfused BATs (see Figures $4(\mathrm{a})-4(\mathrm{c}))$ and only background signals in the unperfused BATs (see Figure 4(d)) was approving the reliable patency of the epigastric artery as central-core vessel (see * at Figure 5) inside the BAT by providing the Luciferin-substrate via blood flow to the BAT. Therefore, this animal model offers both an in vivo bioreactor model for musculoskeletal or other different cell types including a central anastomosable core vessel as well as the possibility of monitoring the transplanted cells, in our study transfected myoblasts in vivo. Thus, the required animal number can be reduced, as there is no exceeding animal sacrifice necessary at different time points. 


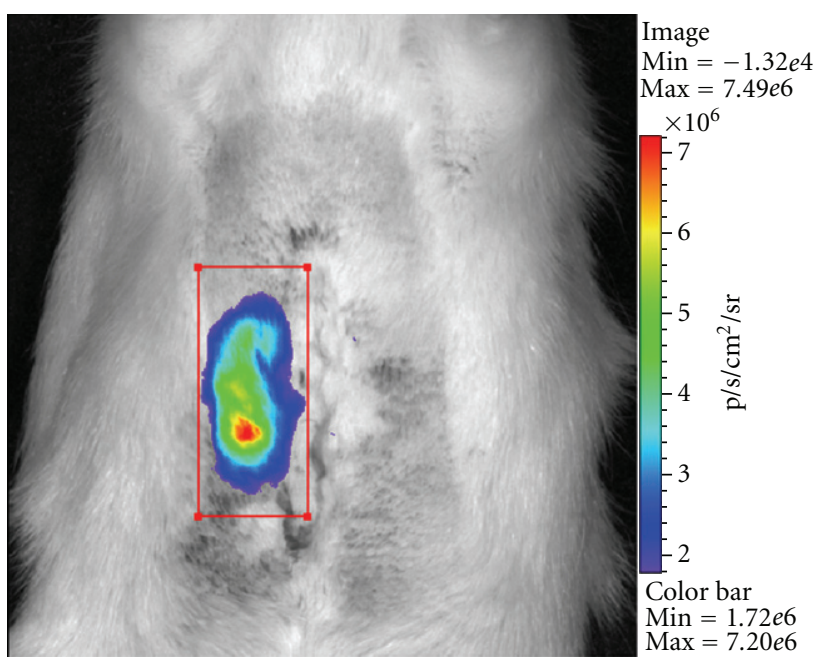

(a)

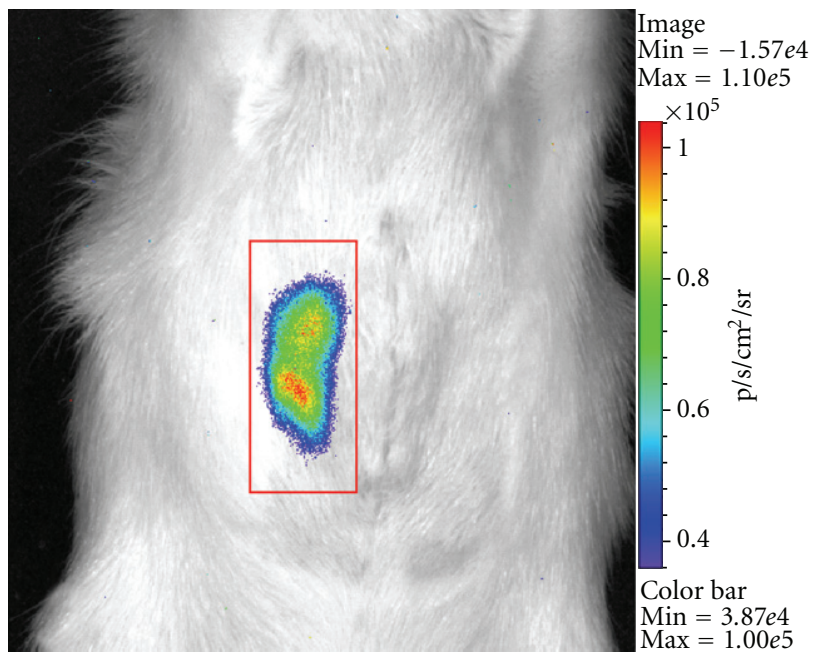

(c)

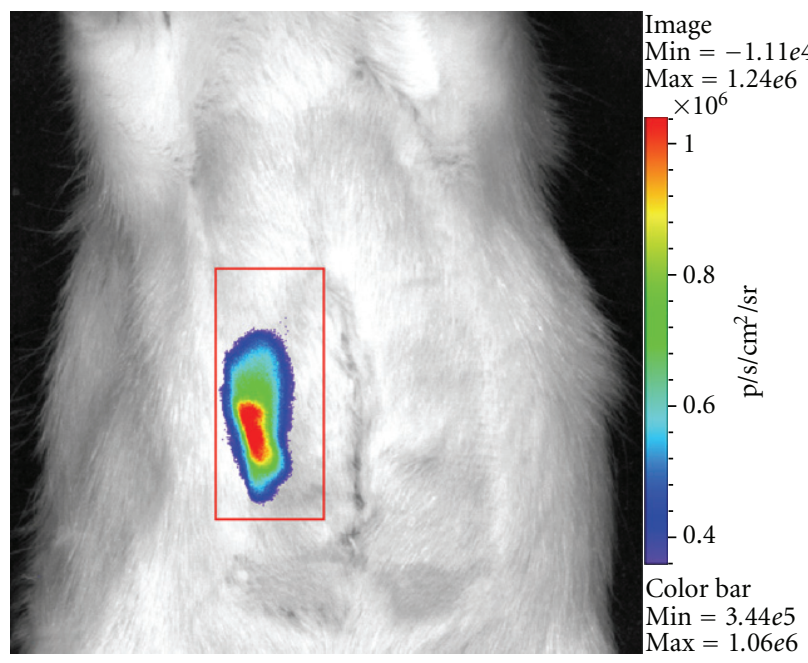

(b)

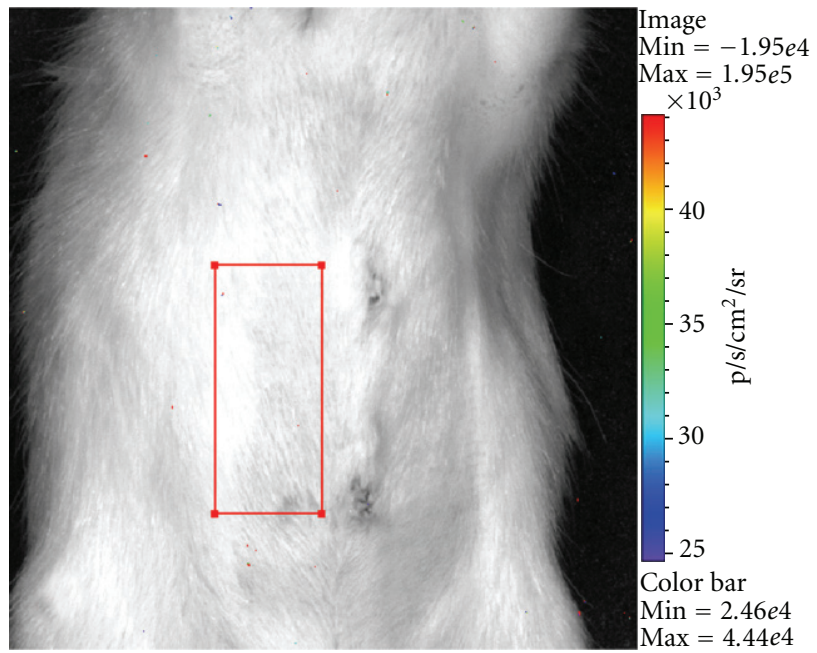

(d)

FIGURE 4: Bioluminescence followup after myoblasts implantation including the epigastric artery as central-core vessel (perfused BAT): (a) day of surgery, (b) 1 day after implantation, (c) 7 days after implantation, (d) 7 days after implantation of myoblasts without central-core vessel in 3D-construct (unperfused BAT).

TABLE 1: Bioluminescence imaging of the BATs perfused $(n=4)$ and unperfused $(n=3)$ on day 1,3 , and 7 after myoblaststransplantation in photons/second and standard deviation (SD). As no substrate (Luciferin) can reach the transfected myoblasts within the unperfused BAT inside the bioreactor chamber, only background signals can be detected.

\begin{tabular}{lcc}
\hline & Perfused BAT & Unperfused BAT \\
\hline & \multicolumn{2}{c}{ photons $/ \mathrm{sec} \pm$ SD } \\
\hline d0 & $1.769 \times 10^{8} \pm 1.576 \times 10^{7}$ & $4.110 \times 10^{5} \pm 3.519 \times 10^{4}$ \\
d1 & $3.426 \times 10^{7} \pm 2.414 \times 10^{6}$ & $5.172 \times 10^{5} \pm 5.822 \times 10^{4}$ \\
d7 & $3.031 \times 10^{6} \pm 4.087 \times 10^{5}$ & $4.652 \times 10^{5} \pm 7.527 \times 10^{4}$ \\
\hline
\end{tabular}

3.2. Capillarization within the Perfused BAT. Pulsatile perfusion within a 3-dimensional bioartificial tissue construct is promoting the capillarization $[8,9]$. In our study, we could show that the epigastric artery is applicable as centralcore vessel. Nevertheless, we could also show that seven days after transplantation there was a beginning capillarization within the BAT, in the areas close to the core vessel (see $<$ at Figure 5). Further we could also prove the presence of myoblasts with positive MyoD staining by immunofluorescence (see Figure 6) after explantation on day seven showing that the myoblast phenotype persisted consistently. However, improvement of the matrix, for example, by inserting synthesized polymer fibers to achieve a more muscle-fiberlike structure will be needed to achieve both, a long-time stability as well as an organized histomorphological pattern 


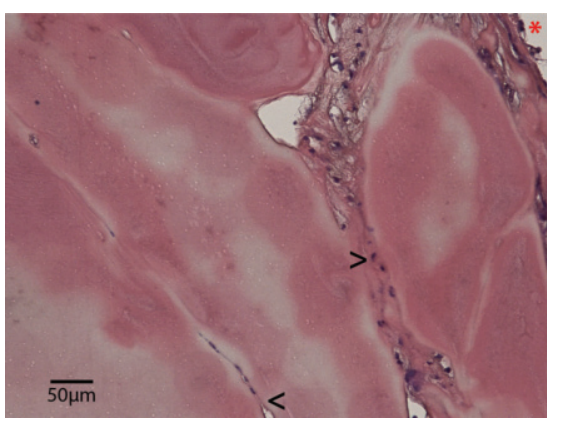

Figure 5: H\&E staining of an explanted perfused BAT seven days after implantation showing the lumen of the epigastric artery $(*)$, myoblasts inside the fibrin matrix $(>)$ as well as starting capillarization within the matrix $(<)$.

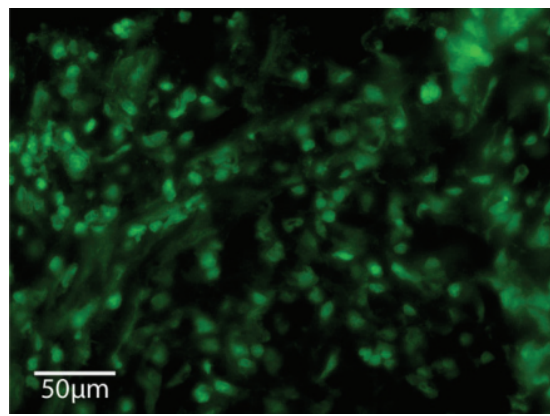

Figure 6: Immunofluorescence, 400x. Explanted perfused BAT seven days after transplantation showing positive MyoD staining for myoblasts.

of the myoblasts to get one more step closer to the use of bioartificial tissue in reconstructive surgery.

\section{Conclusions}

Myoblasts can be transfected with Luciferase and, therefore, bioluminescence is an effective and reliable method for assessment of myoblasts cell graft survival in vivo. The implantation of the in vivo bioreactor with the epigastric artery as core vessel was performed routinely with regular patency of the vessel and without any side effects or wound problems during the experimental period. Again, starting capillarization was found in the areas close to the core vessel. Thus, the epigastric artery is applicable as central-core vessel of the 3-dimensional construct and could later be also used as an anastomosable vessel of artificial musculoskeletal tissue for further experimental reconstructive surgeries after optimizing both matrix and cell survival. However, with the use of a predefined customized in vivo bioreactor chamber, this model offers also a variety of possible applications to create vascularized artificial tissue.

\section{Acknowledgments}

The psPAX2 and pMD2.G plasmids were kindly provided by Didier Trono (LVG-Trono Lab, Lausanne, Switzerland), the lentiviral vector pEMW-Luc by Gan Shu Uin (Department of Surgery, NUS, Singapore). Furthermore, the authors would like to thank Andrea Fritz and Tanja Wollersheim for technical assistance, tissue embedding, processing, and staining as well as Brigitte Kirschner for the bioreactor layout and Nadya Shermain Dunda for the in situ sketch.

\section{References}

[1] H. H. Vandenburgh, "Functional assessment and tissue design of skeletal muscle," Annals of the New York Academy of Sciences, vol. 961, pp. 201-202, 2002.

[2] A. D. Bach, J. P. Beier, J. Stern-Staeter, and R. E. Horch, "Skeletal muscle tissue engineering," Journal of Cellular and Molecular Medicine, vol. 8, no. 4, pp. 413-422, 2004.

[3] M. Koning, M. C. Harmsen, M. J. A. van Luyn, and P. M. N. Werker, "Current opportunities and challenges in skeletal muscle tissue engineering," Journal of Tissue Engineering and Regenerative Medicine, vol. 3, no. 6, pp. 407-415, 2009.

[4] C. A. Rossi, M. Pozzobon, and P. De Coppi, "Advances in musculoskeletal tissue engineering: moving towards therapy," Organogenesis, vol. 6, no. 3, pp. 167-172, 2010.

[5] V. Mudera, A. S. Smith, M. A. Brady, and M. P. Lewis, "The effect of cell density on the maturation and contractile ability of muscle derived cells in a $3 \mathrm{D}$ tissue-engineered skeletal muscle model and determination of the cellular and mechanical stimuli required for the synthesis of a postural phenotype," Journal of Cellular Physiology, vol. 225, no. 3, pp. 646-653, 2010.

[6] M. Radisic, J. Malda, E. Epping, W. Geng, R. Langer, and G. Vunjak-Novakovic, "Oxygen gradients correlate with cell density and cell viability in engineered cardiac tissue," Biotechnology and Bioengineering, vol. 93, no. 2, pp. 332-343, 2006.

[7] J. Koffler, K. Kaufman-Francis, S. Yulia et al., "Improved vascular organization enhances functional integration of engineered skeletal muscle grafts," Proceedings of the National Academy of Sciences of the United States of America, vol. 108, no. 36, pp. 14789-14794, 2011.

[8] Y. Tanaka, K. C. Sung, A. Tsutsumi, S. Ohba, K. Ueda, and W. A. Morrison, "Tissue engineering skin flaps: which vascular carrier, arteriovenous shunt loop or arteriovenous bundle, has more potential for angiogenesis and tissue generation?" Plastic and Reconstructive Surgery, vol. 112, no. 6, pp. 1636-1644, 2003.

[9] T. Kofidis, A. Lenz, J. Boublik et al., "Pulsatile perfusion and cardiomyocyte viability in a solid three-dimensional matrix," Biomaterials, vol. 24, no. 27, pp. 5009-5014, 2003.

[10] R. K. Birla, G. H. Borschel, R. G. Dennis, and D. L. Brown, "Myocardial engineering in vivo: formation and characterization of contractile, vascularized three-dimensional cardiac tissue," Tissue Engineering, vol. 11, no. 5-6, pp. 803-813, 2005.

[11] S. Levenberg, J. Rouwkema, M. Macdonald et al., "Engineering vascularized skeletal muscle tissue," Nature Biotechnology, vol. 23, no. 7, pp. 879-884, 2005.

[12] A. D. Bach, A. Arkudas, J. Tjiawi et al., "A new approach to tissue engineering of vascularized skeletal muscle," Journal of Cellular and Molecular Medicine, vol. 10, no. 3, pp. 716-726, 2006.

[13] E. Polykandriotis, A. Arkudas, R. E. Horch, M. Stürzl, and U. Kneser, "Autonomously vascularized cellular constructs in 
tissue engineering: opening a new perspective for biomedical science," Journal of Cellular and Molecular Medicine, vol. 11, no. 1, pp. 6-20, 2007.

[14] D. Klumpp, R. E. Horch, U. Kneser, and J. P. Beier, "Engineering skeletal muscle tissue-new perspectives in vitro and in vivo," Journal of Cellular and Molecular Medicine, vol. 14, no. 11, pp. 2622-2629, 2010.

[15] L. F. Greer III and A. A. Szalay, "Imaging of light emission from the expression of luciferases in living cells and organisms: a review," Luminescence, vol. 17, no. 1, pp. 43-47, 2002.

[16] A. Sato, B. Klaunberg, and R. Tolwani, "In vivo bioluminescence imaging," Comparative Medicine, vol. 54, no. 6, pp. 631634, 2004.

[17] I. Kutschka, I. Y. Chen, T. Kofidis et al., "Collagen matrices enhance survival of transplanted cardiomyoblasts and contribute to functional improvement of ischemic rat hearts," Circulation, vol. 114, no. 1, pp. I167-I173, 2006.

[18] F. Zhang, W. C. Lineaweaver, S. Kao et al., "Microvascular transfer of the rectus abdominis muscle and myocutaneous flap in rats," Microsurgery, vol. 14, no. 6, pp. 420-423, 1993. 


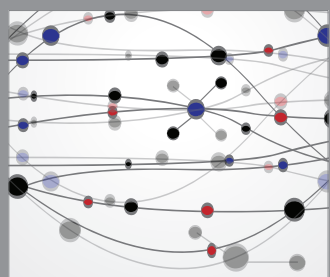

The Scientific World Journal
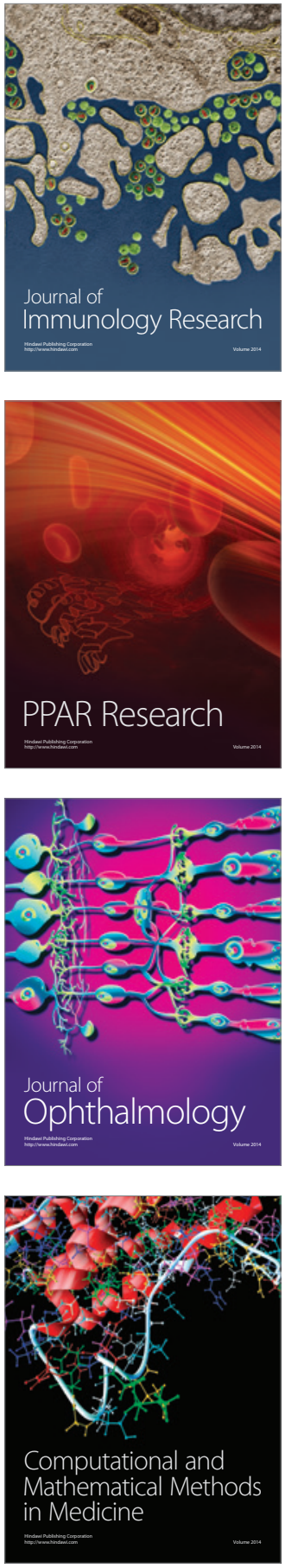

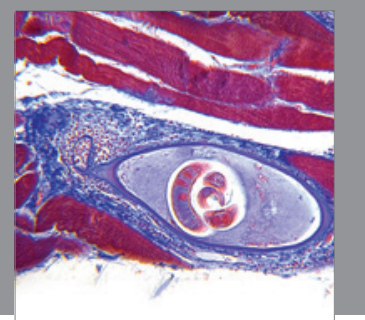

Gastroenterology

Research and Practice
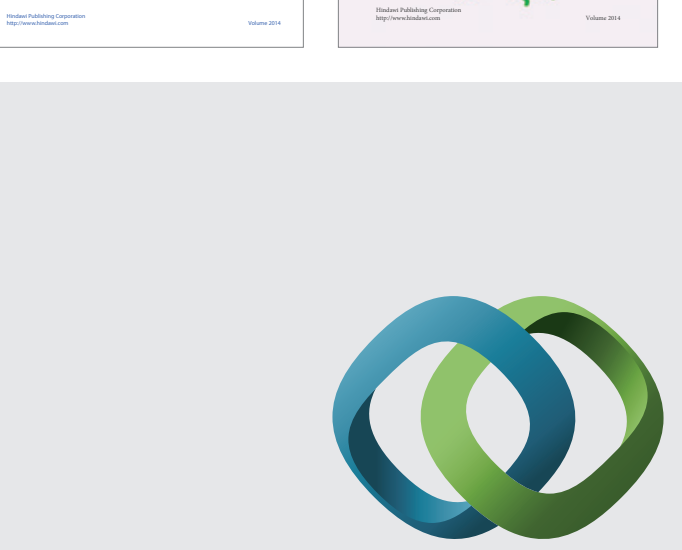

\section{Hindawi}

Submit your manuscripts at

http://www.hindawi.com
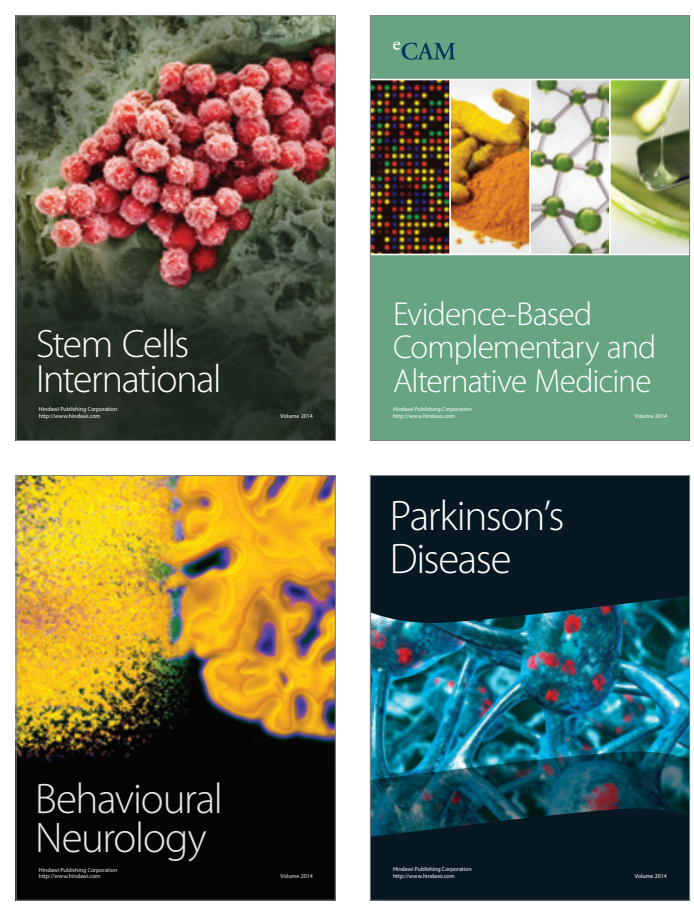

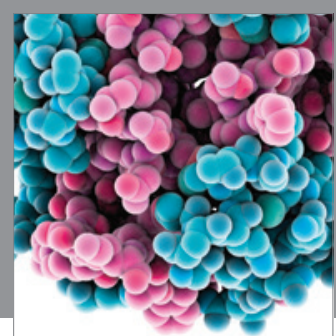

Journal of
Diabetes Research

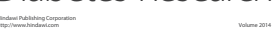

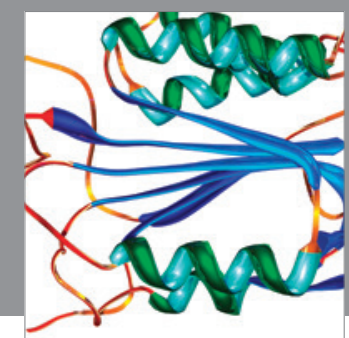

Disease Markers
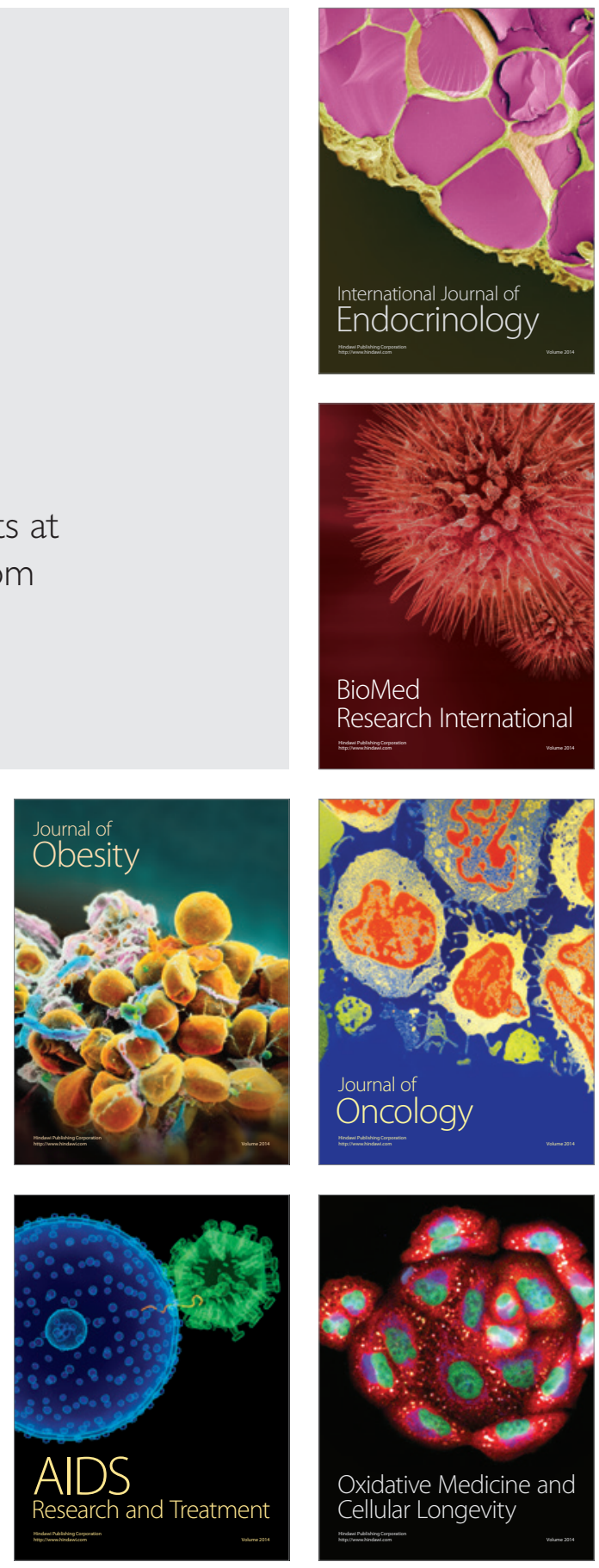